\title{
Medienkompetenz als Schlüssel für Demokratiekompetenz
}

\author{
Michael Schröder
}

\section{$1 \quad$ Einleitung}

Die digitale Transformation der Gesellschaft ist in vollem Gange und macht auch vor den Massenmedien und dem politischen System nicht Halt. Soziale Medien wie Twitter und Facebook sowie Social Bots sind grundsätzlich in der Lage, auch in Wahlkämpfen bestehende Diskussionen zu beeinflussen und neue Themen in Umlauf zu bringen (Jüngling 2015). Im Wahlkampf um die US-Präsidentschaft 2016 wurden soziale Medien und Text-Roboter (Bots) vielfach verwendet und deren mögliche ausländische Herkunft und Einflussnahme diskutiert (Fischer 2016).

Leaks und Desinformationen sind weitere Faktoren, die - durch digitale Medien getrieben - die politische Kommunikation und Willensbildung qualitativ verändern. Leak, der englische Begriff für Leck, Loch oder undichte Stelle, meint die durch anonyme Informantinnen und Informanten (Whistleblower) ermöglichte Veröffentlichung von geheimen Informationen. Berühmt geworden sind die durch die Onlineplattform Wikileaks publizierten Ausschnitte der geheimen Mautverträge mit Toll Collect und Teile der Verhandlungsprotokolle zu ACTA. Die Offshore-Leaks und die Luxemburg-Leaks sind weitere Beispiele, ebenso die Panama und Paradise Papers, die durch einen weltweiten Rechercheverbund von Investigativreportern und -reporterinnen aus 70 Ländern offengelegt wurden. In Deutschland sind die Süddeutsche Zeitung, NDR und WDR Teil des Verbunds.

Fake News - oder besser: die gezielte Desinformation - haben sich in den letzten Jahren - spätestens seit der Präsidentschaft Trumps in den USA - zu

M. Schröder (凶)

Akademie für Politische Bildung, Tutzing, Deutschland

E-Mail: m.schroeder@apb-tutzing.de 
einem politischen Schlagwort entwickelt. Für die wissenschaftliche Diskussion ist dieser Propagandabegriff untauglich. Denn Trump meint damit alle journalistischen Erzeugnisse, die seiner Weltsicht und Programmatik widersprechen. Es ist daher besser, von gezielter Desinformation zu sprechen. Dabei handelt es sich um Nachrichten, die entweder falsch oder irreführend sind und deren Urheber eine manipulative Täuschungsabsicht verfolgen. Nicht gemeint sind versehentliche journalistische Fehler, die im von Zeitdruck geprägten Berufsalltag passieren können, aber nicht sollten. Und wenn, müssen sie so schnell wie möglich von den Redaktionen korrigiert werden.

Diese neuen Phänomene führen bei vielen Mediennutzerinnen und -nutzern zur Verunsicherung. Um die Wirkungsweise und Mechanismen dieser neuen digitalen Medienwelt zu verstehen, ist politische Medienkompetenz gefragt. Dafür müssen Medienbildung und politische Bildung mehr denn je zusammenwachsen.

Medien beeinflussen unser Wissen über die Welt, Gesellschaft, Politik und Wirtschaft. In digitalen Zeiten werden Medien mehr und mehr personalisiert. Politische Informationen werden gemäß Nutzerprofilen selektiert. Zahlreiche Nutzerinnen und Nutzer konsumieren keine News und Meldungen zu politischen Tagesaktualitäten. Das Internet kann wegen der daraus entstehenden digitalen Wissenskluft einer Fragmentierung der Öffentlichkeit und einer medialen Klassengesellschaft Vorschub leisten. Ein mediales Prekariat droht zu entstehen. Politische Bildung im Sinn von Medienkompetenzförderung kann und muss hier ansetzen und gegensteuern. Medienkompetenz kommt eine Schlüsselrolle als Demokratiekompetenz zu. Gefragt sind neues medienkundliches Wissen, neue Fähigkeiten im Umgang mit digitalen Medien, medienkritisches Denken und medienpolitische Urteilsbildung.

\section{Dimensionen von Medienkompetenz}

Bereits zu Beginn der 1970er-Jahre - also lang vor der Vermehrung der elektronischen Programmangebote, der Verbreitung des Internets als Alltagsmedium und der Erfindung von Smartphone und sozialen Medien - hat Baacke (1997) den Begriff der Medienkompetenz geprägt. Er ging davon aus, dass der junge Mensch für eine Orientierung in den komplexen Medienwelten - die damals noch sehr viel überschaubarer waren als heute - neue, zusätzliche Kompetenzen braucht und lernen muss.

Nach Baacke ist Medienkompetenz die Voraussetzung für das angemessene Verständnis und die Entschlüsselung medialer Produkte wie Bild- und Filmsprache. Mehr noch: Baacke geht es um den selbstbestimmten Umgang und aktives 
Handeln mit diesen Produkten. Er spricht dabei von ,,medienbezogener Handlungskompetenz" (Baacke 1999, S. 32). Um nicht im individuellen Bereich stehen zu bleiben, fordert Baacke auch einen ,Diskurs der Informationsgesellschaft“, der ,alle wirtschaftlichen, technischen, sozialen, kulturellen und ästhetischen Probleme“ einbezieht (Baacke 1999, S. 35). Dazu gehört auch eine Einflussnahme auf den Medienmarkt und die Medienpolitik.

Baackes Texte sind nach wie vor aktuell:

Medienkompetenz soll, aufs Ganze gesehen, den Nutzer befähigen, die neuen Möglichkeiten der Informationsverarbeitung souverän handhaben zu können. Auch der humane Fortschritt verläuft heute [...] über elektronische Technologien. Um an ihm teilhaben zu können, benötigen wir alle demnächst nicht nur Anschlüsse, um ans Netz gehen zu können. Wir müssen uns in der computerisierten Medienwelt auch zurechtfinden. Medienkompetenz will genau dies ermöglichen, und insofern umschreibt der Begriff ein durchaus übersichtlich zu machendes Arbeitsfeld, an dessen Bearbeitung Medienpädagogik entscheidend Anteil haben wird. (Baacke 1997, S. 98)

Was er unter Medienkompetenz versteht, beschreibt er so:

Indem wir „Medien“ tionsmedium erfahren, dürfen wir doch nicht davon absehen, dass kommunikative Akte auch in Face-to-face-Situationen, live und in direkter Begegnung, über Sprache und Sprechen, Sich-anschauen, Sich-berühren etc. stattfinden; kurz: Medienkompetenz ist eine Besonderung technisch-elektronisch organisierter Kommunikationsverhältnisse, denen aber andere historisch vorausgehen oder diese eng begleiten [...] Medienkompetenz ist eine Besonderung von „,kommunikativer Kompetenz“ (hier sind alle Sinnesakte der Wahrnehmung gemeint) sowie von „Handlungskompetenz“ (hier sind alle Formen der Weltbemächtigung und Weltveränderung gemeint, die zwar durch kommunikative Akte begleitet werden, aber über diese insofern hinausgehen, als hier Objekte, Gegenstände und Sachverhalte „,verrückt“ werden). „Medienkompetenz“, „kommunikative Kompetenz“ und „Handlungskompetenz“ sind Bausteine, die zusammenzufügen und zu verfugen sind. Allen drei Modalitäten ist in Hinsicht auf „Kompetenz“eines gemeinsam: dass der Mensch ein kompetentes Lebewesen sei [...] Somit ist die Aufgabe der Medienpädagogik, die sich Medienkompetenz nennt, Lernen und Erfahrung zu ermöglichen in Bezug auf Wahrnehmungsweisen der Medien, die „keineswegs schon ins Alltagsrepertoire (gehören)““. (Baacke 1997, S. 98)

Baacke (1999, S. 34) definiert Medienkompetenz über vier Dimensionen, die jeweils Unterdimensionen umfassen:

Da ist zunächst die Fähigkeit zur Medienkritik zu nennen. Sie steht an erster Stelle, weil das vorhandene Wissen über Medien stets reflektiert und erweitert werden sollte. Um die Medienlandschaft $\mathrm{zu}$ analysieren, brauchen Individuen Wissen zum Kontext wie zum Beispiel, dass private Programme weitgehend 
werbefinanziert sind und dies Auswirkungen auf die Programminhalte haben kann. Ebenso sind Kenntnisse über Medienkonzentrationen nötig. Nur damit können problematische Medienentwicklungen kritisch und differenziert betrachtet werden. Jeder Mensch sollte sein Wissen aber auch auf sich selbst beziehen können und sein eigenes (Medien-)Handeln kritisch reflektieren. Die Analyse der Medienentwicklungen und der reflexive Rückbezug auf das eigene Handeln können schließlich auch daraufhin abgestimmt werden, ob sie sozial verantwortlich sind. So erhält die Medienkritik auch eine ethische Dimension.

Neben sie tritt die Medienkunde, die das Wissen über heutige Medien und Mediensysteme meint. Auf einer informativen Ebene sind dies klassische Wissensbestände wie z. B. Kenntnisse über die Struktur des Rundfunksystems, die journalistische Arbeitsweise oder auch die Möglichkeiten, einen Computer effektiv für persönliche Zwecke zu nutzen.

Mit Mediennutzung ist zunächst der rezeptiv-anwendende Bereich der Mediennutzung gemeint, also die Nutzungs- bzw. Rezeptionskompetenz jedes einzelnen Menschen im Umgang mit Medien. In diesem Sinne wird Fernsehen oder die Nutzung des Internets z. B. als aktive Tätigkeit gesehen, die kompetent gestaltet werden soll und muss. Die zweite Unterdimension der Mediennutzung ist der Bereich auffordernden Anbietens und interaktiven Handelns. Es gibt heute eine Vielzahl von Möglichkeiten (Online-shopping, Onlinebanking, Produktion von Podcasts, Fotos und Filmen), um auf den Plattformen in der digitalen Medienwelt interaktiv tätig zu sein (Instagram, YouTube).

Der letzte Bereich ist derjenige der Mediengestaltung. Medien verändern sich ständig und es steht jedem frei, neue Inhalte gestaltend einzubringen. Wer kompetent mit Medien umgeht, kann sie kreativ mitgestalten und innovativ weiterentwickeln. Er/sie bringt sich ein mit ästhetischen Varianten und überschreitet damit die Grenzen bestehender Kommunikationsroutinen.

Mehr denn je hat Medienkompetenz unter den Bedingungen des digitalen Zeitalters eine herausragende Bedeutung. Dazu gehört, dass gerade Kinder und Jugendliche lernen, sich bewusst und kritisch im Netz zu bewegen und es aktiv und selbstbestimmt für die eigenen Zwecke zu nutzen. Dazu gehört ein gewisses Verständnis, wie Algorithmen funktionieren und wie sie die abgefragten und genutzten Inhalte des Netzes beeinflussen. Ebenfalls unentbehrlich ist ein Grundwissen darüber, wie das Netz funktioniert. Wem gehören die großen USInternetkonzerne und wie sind sie untereinander verbunden? Die Nutzer und Nutzerinnen müssen lernen: Was passiert mit meinen Daten? Wie kann ich sie vor unerlaubtem Zugriff und Missbrauch bewahren? Wie schütze ich mich vor Mobbing in „sozialen“ Netzwerken? Es reicht in Zukunft nicht mehr aus, sich halbwegs gekonnt an der Oberfläche des Netzes zu bewegen und Dienste wie 
E-Mails, Suchmaschinen wie Google und „soziale“ Netzwerke wie Facebook, Twitter und Instagram zu nutzen. Letztendlich muss es bei digitaler Medienkompetenz auch darum gehen, die Grundzüge der Technik zu verstehen, um nicht eines Tages von ihr beherrscht und kontrolliert zu werden.

\section{Demokratiekompetenz als Ziel politischer Bildung}

Die digitale Transformation betrifft auch das politische System. Durch das digitale Onlinezeitalter haben sich die politischen Informationsangebote sowie die Möglichkeiten, selbst politisch aktiv zu werden und die öffentliche Willensbildung mitzugestalten, grundlegend und qualitativ verändert.

Noch vor Beginn der digitalen Transformation, die alle gesellschaftlichen Bereiche erfasste, hat im Jahr 1995 eine Gruppe namhafter Vertreter und Vertreterinnen der politischen Bildung den „Darmstädter Appell“ veröffentlicht. Dessen Kernaussagen haben trotz veränderter Rahmenbedingungen nichts von ihrer Gültigkeit verloren: Ziel politischer Bildungsarbeit müsse die Befähigung der Schülerinnen und Schüler zur Wahrnehmung ihrer Bürgerrolle in der Demokratie sein. Zur Ausfüllung dieser Rolle benötigten die Menschen verschiedene Kompetenzen: Wissen über das Gesellschafts- und vor allem das politische System und seine Institutionen, über den Ablauf politischer Prozesse und die dabei geltenden Regeln. Ferner gehörten dazu bestimmte Einstellungen und Verhaltensdispositionen. Weiter würden Fähigkeiten wie Handlungs- und Gestaltungskompetenz zur Nutzung von Partizipationschancen sowie eine auf Politik bezogene Entscheidungs- und Problemlösungsfähigkeit benötigt (Darmstädter Appell 1995, S. 35).

Ähnlich hat es das „Münchner Manifest“ mit dem Titel „Demokratie braucht politische Bildung“ im Mai 1997 formuliert. Autorinnen und Autoren sind die Leiter und Leiterinnen der Bundeszentrale und der Landeszentralen für politische Bildung. Es heißt dort, dass der demokratische Rechtsstaat vom mündigen Mitdenken und Mittun seiner Bürgerinnen und Bürger sowie von ihrer Bereitschaft lebe, sich selbstverantwortlich und sozialverantwortlich ein Urteil zu bilden, in der Verfassung normierte Regeln und Werte zu respektieren und sich für sie zu engagieren. Die durch gesellschaftliche Umbrüche gekennzeichnete Gegenwart fordere die Demokratiekompetenz der Bürgerinnen und Bürger auf besondere Weise heraus. Nur eine Bürgerschaft, die auf qualifizierte Weise am Zustandekommen politischer Entscheidungen teilhabe, stehe auch in Zeiten gesellschaftlicher 
Umbrüche zur Demokratie. Politische Bildung arbeite für eine aktive Bürgergesellschaft, die den Staat als Summe aller Bürgerinnen und Bürger begreife und nicht als dienstleistendes Gegenüber (Münchner Manifest 1997, S. 37-38).

Zusammenfassend lässt sich sagen, dass das Ziel der Bemühungen politischer Bildung der mündige Bürger bzw. die mündige Bürgerin ist. Als solche kennen sie die Strukturen des politischen Systems und ihre Beteiligungsrechte. Sie sind fähig, rational zu urteilen und aktiv in die Politik einzugreifen. Diese Ansprüche sind hoch und bedürfen der Anstrengung aller. Joachim Detjen (2000) bringt die von der Wertewandelforschung inspirierte Forschung zu Persönlichkeitstypen unter dem Blickwinkel ihres politischen Engagements in folgende Ordnung:

1. Desinteressierte,

2. reflektierte Zuschauerinnen und Zuschauer,

3. interventionsfähige Bürgerinnen und Bürger

4. Aktivbürgerinnen und -bürger.

Die Desinteressierten bezeichnet er als „die perfekten Privatiers“. (Detjen 2000, S. 19) Diese zahlenmäßig nicht unerhebliche Bevölkerungsgruppe nimmt für sich das Recht in Anspruch, sich um Politik nicht zu kümmern und von ihr in Ruhe gelassen zu werden. Die reflektierten Zuschauerinnen und Zuschauer sind wohl die größte Gruppe. Sie mischen sich zwar nicht in die Politik ein, informieren sich aber über politische Ereignisse und Themen. Sie sprechen im persönlichen Umfeld über Politik. Sie gehen zur Wahl und beteiligen sich an Abstimmungen. Die interventionsfähigen Bürgerinnen und Bürger zeigen zwar kein dauerhaftes politisches Engagement, sind aber fähig und bereit, je nach Situation aktiv in die Politik einzugreifen. Die Aktivbürgerinnen und -bürger sind die kleinste Gruppe. Das Politische nimmt bei ihnen einen sehr hohen Stellenwert ein. Die Angehörigen dieser Gruppe haben sich zur Mitgliedschaft und zur Mitarbeit in Parteien, Interessenverbänden oder Vereinigungen entschlossen. Aus dieser Gruppe rekrutiert sich auch das politische Führungspersonal eines Gemeinwesens. Detjen rät der politischen Bildung, bei ihrer Zieldefinition auf diese vier Gruppen Rücksicht zu nehmen:

Sie sollte den reflektierten Zuschauer als ihr Minimalziel ansehen. Als anspruchsvolleres, aber wohl doch realistisches Regelziel sollte sie den interventionsfähigen Bürger betrachten. Den Aktivbürger sollte sie keinesfalls aus den Augen verlieren, ihn sogar als Maximalziel ihrer Arbeit betrachten, aber nicht vergessen, dass nur die wenigsten diesen Grad an Bürgerschaftlichkeit erreichen. Die Desinteressierten schließlich bilden eine ständige Herausforderung für die Bildungsbemühung. Ihre Zahl zu verringern und aus Desinteressierten wenigstens im Ansatz reflektierte Zuschauer zu machen, ist 
der politischen Bildung dauerhaft aufgegeben. Sie darf sich nicht entmutigen lassen, auch wenn ihre Erfolge auf diesem Feld nur sehr bescheiden sind. (Detjen 2000, S. 19)

Darüber hinaus entfaltet Joachim Detjen unter Bezugnahme auf Buchstein und Münkler den Begriff der bürgerschaftlichen Kompetenz, die sich in kognitive, prozedurale und habituelle Kompetenzen entfaltet (Detjen 2000, S. 12-13):

Mit der kognitiven Kompetenz ist ein gewisses Niveau an Wissen und Lernfähigkeit gemeint. Dieses Wissen bezieht sich auf die institutionelle Ordnung des politischen Systems, auf funktionale Zusammenhänge innerhalb dieses Systems bis hin zu seinen weltpolitischen und -wirtschaftlichen Abhängigkeiten. Bürgerinnen und Bürger sollen aber auch hinsichtlich der Inhalte der aktuellen politischen Entscheidungen über sachlich informierte Kenntnisse verfügen.

Prozedurale Kompetenzen sind Kenntnisse und Fertigkeiten, die benötigt werden, um politische Einflussmöglichkeiten und Partizipationschancen auch tatsächlich wahrnehmen und nutzen zu können. Bürgerinnen und Bürger müssen deshalb Kenntnisse über administrative Zuständigkeiten und rechtliche Verfahren besitzen. Sie müssen über strategische Fähigkeiten verfügen, um die eigenen oder als richtig erkannten allgemeinen Ziele verwirklichen zu können.

Habituelle Kompetenzen sind Einstellungen, die Bürgerinnen und Bürger dem Staat zu dessen Bestandserhaltung entgegenbringen müssen. Sie müssen affektiv fest verankert sein, damit sie handlungsmotivierend wirken können. Moderne Demokratien sind in ihren habituellen Erwartungen besonders anspruchsvoll. Als staatliche Gemeinwesen benötigen sie natürlich Rechtsgehorsam, darüber hinaus aber auch Opferbereitschaft, das Gemeinwesen gegen Bedrohungen von außen zu verteidigen. Als liberale Gemeinwesen bedürfen sie der Fairness und der Toleranz in weltanschaulichen Angelegenheiten. Als demokratische Gemeinwesen sind sie angewiesen auf Partizipation, die nach Möglichkeit rational, verantwortbar und regelmäßig sein soll. Als sozialstaatliche Gemeinwesen kommen sie nicht ohne sozialen Gerechtigkeitssinn und Solidarität aus.

An dieser Stelle sei darauf hingewiesen, dass mündige Bürgerinnen und Bürgern in einer digitalen Welt über Medienkompetenz verfügen müssen. Hilfreich scheint dabei, dass Ziele der Medienkompetenzförderung auch in Zielen politischer Bildung enthalten sind:

Die betont ihrerseits Kenntnisse, Kommunikations-, Urteils- und Handlungsfähigkeiten sowie -bereitschaften. Siehe dazu auch GPJE (2004), Detjen et al. (2012), Kühberger (2009) und Krammer (2008).

Gapski (2017) unterscheidet digitale Demokratiekompetenz in (1) Kompetenzen für die Anwendung digitaler Technologien im Kontext inhaltlich-politischen 
und demokratierelevanten Handelns und Denkens (gefördert bspw. durch AntiHate-Speech-Projekte) und (2) Kompetenzen für die Erschließung und Hinterfragung digital vermittelter Welt- und Selbstbezüge (gefördert bspw. durch Reflexion über Privatheit, Souveränität usw.). Für den vorliegenden Zusammenhang ist vor allem der zweite Aspekt wichtig.

Die digitale Transformation durchdringt nahezu jede Kommunikation und damit alle gesellschaftlichen Bereiche. Treibende Kraft ist die digitale Ökonomisierung der Kommunikation. Durch die wirtschaftlich vorangetriebene Datafizierung der Gesellschaft entstehen neue Machtpole und Informationsasymmetrien. Diese neuen Strukturen sind je schon politisch in ihren Auswirkungen (Privatheit/Öffentlichkeit, Diskriminierungen usw.) und müssen damit zum Thema und Inhalt der Demokratiekompetenzförderung werden.

Die Forderung nach digitaler Demokratiekompetenz wird als Fortsetzung der Diskussion über „Medienkompetenz“ im analogen Zeitalter gesehen. Sie betont insbesondere die politische Partizipation und bringt neue, bislang unbearbeitete und unbekannte Herausforderungen durch die digitale Transformation hervor. Die ,alte“ Medienkompetenz für die analoge Mediengesellschaft unterscheidet sich von der neuen digitalen Demokratiekompetenz durch ihre Technologien wie das Internet, künstliche Intelligenz, Algorithmen und Big Data, sowie durch den Einbezug aller als Produzenten und Konsumenten, die sogenannten „Prosumer“. Digitale Demokratiekompetenz muss also auch ein Wissen über das Zusammenspiel ökonomischer und technologischer Treiber beinhalten. Darüber hinaus ist von der Idee Abstand zu nehmen, dass ein handlungsfähiges, selbstbestimmtes Subjekt die digitale Durchdringung der Gesellschaft alleine verstehen kann. Durch die kritische Reflexion und Maßnahmen zur „digitalen Selbstverteidigung“ allein kann auch das digitalkompetente Individuum - wenn es denn überhaupt real existiert - keine umfassende Souveränität in der digitalen Welt erlangen. Ist also die ohnmächtige Hinnahme eines Lebens unter den Bedingungen des Kontrollverlusts über die eigenen Daten die unausweichliche Konsequenz? Dies kann für Politische Bildung im demokratischen System nicht hinnehmbar sein. Deswegen brauchen wir eine konzertierte Aktion aller Beteiligter, um den Kontrollverlust (vgl. Seemann 2014) einzudämmen. Damit sind gemeint: die Nutzerinnen und Nutzer selbst, die Internetkonzerne sowie die zivilgesellschaftlichen und politischen Akteurinnen und Akteure. Voraussetzung ist die Gestaltung geeigneter rechtlicher, technischer und politischer Rahmenbedingungen und Regulierungen. Denn selbst die utopische Konstruktion eines digitalkompetenten Individuums ist mit diesen Aufgaben allein überfordert. Deshalb ist es auch ein Ziel politischer 
Demokratiebildung, ein Bewusstsein für die Bedeutung dieser Rahmenbedingungen und die ihnen zugrundeliegende Werte und Ethiken zu schaffen. Andererseits sind Demokratiekompetenzen gefordert.

Politische Bildung über Politik findet unter den Bedingungen einer digitalen Mediengesellschaft statt. Es geht nicht nur um Information über die mediale „Darstellung“ von Politik, sondern auch die vertiefende Auseinandersetzung mit den medienabhängigen und -unabhängigen Faktoren der „Herstellung“ von Politik. Medien interessieren deshalb als Agenturen der Politikvermittlung. Insgesamt müssen Medien als eine Dimension des Politischen bzw. auch Unpolitischen in den Blick kommen.

\section{$4 \quad$ Veränderungen der Medienlandschaft und -nutzung}

Die Medienlandschaft und die Nutzung haben sich in den letzten Jahren deutlich verändert. So ist die tägliche TV-Sehdauer in der deutschen Gesamtbevölkerung von durchschnittlich $223 \mathrm{~min}$ im Jahr 2010 auf $211 \mathrm{~min}$ im Jahr 2019 zurückgegangen. Besonders deutlich war der Rückgang in der Gruppe der Kinder von 3 Jahren bis 13 Jahren: Von 93 (2010) auf $58 \mathrm{~min}$ (2019). Ein Zuwachs ist lediglich bei der älteren Bevölkerung ab 70 festzustellen (Media Perspektiven Basisdaten 2019, S. 70, 72).

Genau gegenläufig entwickelt sich die Nutzung des Internet: Waren 2017 noch $59 \%$ der Gesamtbevölkerung täglich online, sind es 2019 bereits $71 \%$. Bei der täglichen Nutzung in Minuten ist der Zuwachs in den letzten zwei Jahren bei den 14- bis 29-Jährigen mit plus 15 min auf 201 min täglich (2019) besonders hoch. Der Anteil der Offlinerinnen und Offliner geht kontinuierlich zurück und ist bei den über 60-Jährigen mit 30,4 \% besonders hoch (Media Perspektiven Basisdaten 2019, S. 81-85).

Die MedienGewichtungsstudie 2019-II der deutschen Landesmedienanstalten, die für die Aufsicht des privaten, kommerziellen Rundfunks zuständig sind, bestätigt diese Daten: Beim Meinungsbildungsgewicht gewinnt das Internet weiter an Bedeutung (eine Verdoppelung von $13 \%$ in 2009 auf jetzt knapp $30 \%$ in 2019). Die Tageszeitung verliert kontinuierlich und fällt erstmals unter $18 \% .2009$ lag sie noch bei $26 \%$. Das Fernsehen kann seinen Spitzenplatz mit $32 \%$ zwar noch halten, verlor in den vergangenen zehn Jahren aber sechs Prozent. Radio (18\%) und Zeitschriften (zwei Prozent) zeigen über die Jahre hinweg ein nahezu ähnliches, fast unverändertes Meinungsbildungsgewicht.

Die informierende Onlinenutzung stieg bei den 14- bis 29-Jährigen weiter an und liegt mittlerweile bei knapp $73 \%$. Nur in der Generation der über 50-Jährigen 
bleibt Fernsehen trotz Rückgang das meistgenutzte Informationsmedium. In der Gesamtbevölkerung hat das Internet mit knapp $36 \%$ der Nennungen mittlerweile das Fernsehen $(31 \%)$ als das wichtigste Informationsmedium überholt. Die Tageszeitung, das Radio und Zeitschriften sind deutlich weniger relevant (Landesmedienanstalten 2019).

Die Rolle des professionellen Journalismus der klassischen, meist analogen Massenmedien als beherrschender Faktor der öffentlichen Meinungsbildung hat sich seit dem Aufkommen des Internets und insbesondere in den sozialen Netzwerken grundlegend gewandelt. Er hat seine fast monopolartige Funktion und Bedeutung als Schleusenwärter im täglichen Nachrichtenstrom verloren. Die klassischen Nachrichtenmedien sind heute nur noch ein Player unter vielen im globalen Angebot des Netzes. Die Zeiten, in denen Nachrichten ihren Weg durch die Filter der Redaktionen ans Licht der Öffentlichkeit finden mussten, um wahrgenommen zu werden, sind endgültig vorbei. In Zeiten von Twitter und Facebook braucht ein Politiker keine Pressekonferenz mehr, um sich über den journalistischen Umweg Gehör und Aufmerksamkeit beim Publikum zu verschaffen. Er kommt direkt und in Echtzeit mit seiner Weltsicht und seinen Meinungen zu den Wählerinnen und Wählern (vgl. dazu Lobigs und Neuberger 2018; Wallace 2018).

Das soll nicht heißen, dass die professionellen Nachrichtenmedien ihre Bedeutung völlig verloren haben. Für viele Menschen sind sie immer noch und nach wie vor wichtige Informationsquellen, aber eben nicht mehr die einzigen: $85 \%$ der deutschen Haushalte werden noch vom Fernsehen erreicht. Die Hauptnachrichtensendung der ARD, die Tagesschau um 20 Uhr wird im Durchschnitt täglich von 9,6 Mio. Menschen gesehen; die ZDF-Nachrichten um 19 Uhr sehen etwas über 4 Mio.. 53 Mio. hören täglich Radio. Und die deutschen Tageszeitungen lesen täglich rund 40 Mio. Menschen (Media Perspektiven 2019, S. 70-80).

Neben diese klassischen Informationsmedien ist ein bunter Infococktail getreten - analog und digital - den sich die Bürgerinnen und Bürger täglich neu zusammenmixen. Der professionelle Journalismus steht in Konkurrenz mit alternativen Angeboten auf sozialen Netzwerken und Formen öffentlicher Bürgerkommunikation. Auch dort entstehen Meinungsaustausch und Meinungsklima. Eine nicht zu unterschätzende Rolle spielen auch Suchmaschinen, die mit ihrem Algorithmus personalisierte Nachrichtenkanäle bereitstellen und die Nutzerinnen und Nutzer zu Seiten führen, die ihren Neigungen und Voreinstellungen entsprechen (Schweiger et al. 2019). Wolfgang Schweigers These ist folglich zuzustimmen: „Wir leben tatsächlich in einer Zeit des Aufstiegs sozialer Medien und des Bedeutungsverlusts journalistischer Nachrichten“ (2017, S. 182).

Besonders anfällig für diese neuen Formen alternativer, öffentlicher Bürgerkommunikation ist die von Schweiger sogenannte politisierte Bildungsmitte: „Das 
sind Menschen mit niedriger, häufiger noch durchschnittlicher formaler Bildung. Sie sind überwiegend mittleren Alters, informieren sich intensiv im Internet und diskutieren dort auch teilweise mit" (2017, S. 182).

In dieser Gruppe hat sich in den vergangenen Jahren eine deutliche Politisierung und Polarisierung bemerkbar gemacht. Festzumachen sind Fixpunkte wie die Griechenland-Hilfen der EU in der Folge der Eurokrise, Islamismus und Flüchtlinge. Viele Menschen in dieser Gruppe - aber nicht nur sie - haben sich von den etablierten "Systemmedien“ abgewandt. Hauptinformationsquellen sind das unterhaltungsorientierte und boulevardeske kommerzielle Fernsehen sowie alternative Onlinemedien. Der Austausch mit Gleichgesinnten im Netz - ebenso Eliten- und Politikverdrossene wie sie selbst - fördert und bestärkt das vorhandene Meinungsklima.

An den analogen Stammtischen der Vergangenheit hat es immer schon einzelne, abweichende oder abseitige Meinungen bis hin zum Extremismus gegeben. Damals ging man vom Stammtisch nach Hause und der Neonazi blieb dort allein mit seinen Ansichten. Er erzielte über seinen Stammtisch hinaus keine Wirkung. Jetzt, im digitalen Zeitalter vernetzter Onlinekommunikation, geht er nach Hause und teilt seine extremistischen Ansichten online über soziale Medien und findet schnell Gleichgesinnte. Und es entsteht das Gefühl, vermeintlich einer Mehrheit anzugehören. Dabei ist das Klima in diesen Foren deutlich rauer geworden.

Die Veränderung des Informations- und Diskussionsverhaltens durch das Internet führt auch dazu, dass viele Menschen sich für besser informiert halten als sie es tatsächlich sind. Schweiger sieht eine „merkwürdige Mischung aus politischer Aufwallung, mangelnder Medienkompetenz, einem fast schon übernatürlichen politischen Selbstbewusstsein bei gleichzeitiger Fehlinformiertheit" (2017, S. 183). Eine Ursache dafür ist im Bedeutungsverlust des professionellen Journalismus zu suchen (siehe oben). Bei aller berechtigter Kritik an Fehlleistungen: Nur mithilfe eines freien und unabhängigen Journalismus, wie er insbesondere bei den öffentlich-rechtlichen Rundfunkanstalten, Tageszeitungen, Magazinen und Wochenzeitungen praktiziert und gepflegt wird, ist ein qualifizierter Dialog zwischen den Mitgliedern einer demokratischen Gesellschaft zu organisieren. Und dazu muss die regelmäßige und kompetente Mediennutzung treten, um zu einem soliden politischen Grundwissen, pluralistischer Informiertheit und einer realistischen Einschätzung des Meinungsklimas zu kommen.

Denn je mehr sich Mediennutzerinnen und -nutzer auf die Auswahl beschränken, die ihnen von Nachrichtenaggregatoren wie Google, Twitter oder Facebook personalisiert geliefert wird, umso größer ist die Gefahr der Desinformation. Hier ist das Risiko, sich nur noch in Filterblasen und Echokammern zu bewegen, besonders groß. Es fehlt die professionelle Instanz, die einordnet, gewichtet, 
erklärt und hinterfragt. Auch wenn dabei journalistische Inhalte genutzt werden: Die eigentlichen Quellen sind nicht mehr unbedingt und zweifelsfrei nachvollziehbar. Außerdem bleibt das Problem, dass Nachrichtenmedien die von ihnen in sozialen Netzwerken geposteten Beiträge nicht unbedingt und in erster Linie nach gesellschaftlicher Relevanz aussuchen, sondern nach zu erwartenden möglichst hohen Klickzahlen, Likes und Retweets.

In den entstehenden Filterblasen und Echokammern bleiben die unterschiedlichen Meinungslager unter sich. In diesen homogenen Netzwerken kommen Andersdenkende kaum vor und die Wahrscheinlichkeit, auf entgegengesetzte Meinungen und kontroverse Ansichten zu stoßen, ist eher gering. So bleibt auf der Strecke, was für einen offenen Diskurs in einem demokratisch-pluralistischen Gemeinwesen unentbehrlich ist: die kritische Prüfung der eigenen Positionen und von anderen Denkweisen lernen.

Mittlerweile haben einige Kommunikationswissenschaftler und wissenschaftlerinnen Vorbehalte gegenüber diesem Modell geäußert. So argumentiert Axel Bruns, dass der Einfluss von Echokammern und Filterblasen stark überbewertet werde. Er ergebe sich aus einer moralisierenden und panischen Einstellung zur Rolle von sozialen Medien. Es existiere eine weit verbreitete Tendenz, Plattformen und ihre Algorithmen für politische Polarisierung verantwortlich zu machen. So würden weitaus ernstere Probleme im Zusammenhang mit dem Aufkommen von Populismus verschleiert (Bruns 2019).

Elizabeth Dubois und Grant Blank haben bei einer Untersuchung in Großbritannien herausgefunden, dass diejenigen, die sich für Politik interessieren und einen vielfältigen Medienkonsum haben, dazu neigen, Echokammern zu meiden. Wahrscheinlich würde sich nur ein kleiner Teil der Bevölkerung in einer Echokammer befinden. Nichtsdestotrotz bleibt festzuhalten, dass es Gruppen in der Gesellschaft gibt, die ein verfestigtes, populistisches oder gar extremistisches Bild von Demokratie und Gesellschaft haben und die sich deshalb gerne in Filterblasen und Echokammern aufhalten (Dubois und Blank 2018).

Menschen in diesen Filterblasen, die von den algorithmisch gesteuerten Suchmaschinen und sozialen Medien in ihrem Meinungsbild geprägt werden, haben kaum eine Chance, zu mündigen, demokratiekompetenten Bürgerinnen und Bürgern im oben beschriebenen Sinn zu werden. Die Wahlergebnisse der letzten Jahre in Deutschland haben die rechtspopulistische Alternative für Deutschland in alle Landesparlamente und in den Bundestag gebracht. Dies ist ein Indiz dafür, dass die Polarisierung der Gesellschaft zugenommen hat. Die ehemaligen großen Volksparteien CDU/CSU und SPD, die noch in den 1970er-Jahren bei Wahlen zusammen rund $90 \%$ der Stimmen auf sich vereinigen konnten, schaffen bei aktuellen Umfragen gerade noch knapp über $50 \%$. Bei sieben Parteien und sechs 
Fraktionen im Deutschen Bundestag wird eine Regierungsbildung immer schwieriger. Auch diese Zerrissenheit des politischen Spektrums und Polarisierung ist eine Folge der oben beschriebenen veränderten Mediennutzung und dem damit zusammenhängenden Informations- und Diskussionsverhaltens eines großen Teils der Bevölkerung.

Die neueste Mitte-Studie der Friedrich-Ebert-Stiftung und einem Forschungsteam des Instituts für Interdisziplinäre Konflikt- und Gewaltforschung der Universität Bielefeld (IKG) vom April 2019 stützt diesen Befund. Es ergebe sich das Bild einer Verfestigung und Normalisierung rechter Einstellungen in der Mitte der Gesellschaft, so die Autoren und die Autorin der Studie (Friedrich-Ebert-Stiftung 2019).

Die Studienreihe gibt seit 2006 Auskunft über die Stabilität und Instabilität der Demokratie. Die aktuellen Ergebnisse machen deutlich:

Der Großteil der Deutschen befürwortet die Demokratie, begrüßt die Vielfalt der Gesellschaft und fordert eine Stärkung der EU. Doch zugleich äußert ein Drittel auch nicht-liberale Einstellungen zur Demokratie, stellt gleiche Rechte für alle infrage. Dabei ist die Zustimmung zu menschenfeindlichen Vorurteilen in denen letzten fünf Jahren nahezu unverändert. Das gilt vor allem für Abwertungen gegenüber Zugewanderten, Musliminnen und Muslimen und für Antisemitismus, die seit 2014 hoch sind (Friedrich-Ebert-Stiftung 2019).

Negative Einstellungen gegenüber Asylsuchenden haben sogar zugenommen: Jede zweite befragte Person stimmt negativen Meinungen gegenüber Asylsuchenden zu. Dies ist noch einmal im Vergleich zu 2016 angestiegen, obwohl die Zahl der Asylsuchenden im Befragungszeitraum rückläufig ist. [...] Auch Verschwörungsmythen finden generell in der Bevölkerung großen Zuspruch. $46 \%$ meinen, geheime Organisationen würden politische Entscheidungen beeinflussen. [...] Nahezu ein Viertel der Befragten mutmaßt, Medien und Politik steckten unter einer Decke. (Friedrich-Ebert-Stiftung 2019)

Die Studie kommt zu folgendem Schluss: „Vordergründig findet sich eine hohe Zustimmung zur Demokratie, die aber zugleich von antidemokratischen und antipluralistischen Überzeugungen begleitet wird. Die Mitte verliert ihren festen Boden und ihre demokratische Orientierung." (Friedrich-Ebert-Stiftung 2019)

\section{$5 \quad$ Die Janusköpfigkeit der digitalen Onlinewelten}

Die bislang erfolgten Analysen und Schlussfolgerungen sollen nun aber nicht dazu führen, die Möglichkeiten des Internets und der digitalen Kommunikation in Bausch und Bogen zu verdammen. Wir haben es durchaus mit zwei Seiten 
einer Medaille zu tun. Denn zweifelsfrei bietet das Netz neue Informationsmöglichkeiten, die in der analogen Welt unbekannt waren:

Überall auf der Welt sind - oft in Echtzeit und aktuell - Informationen aus unzähligen Quellen verfügbar. Die Kosten sind für die Nutzerinnen und Nutzer meist überschaubar oder liegen bei null - wenn man von der Überlassung persönlicher Daten einmal absieht, mit denen die Internetkonzerne Geld und Macht kumulieren. Bill Gates' Vision von der „Information at your fingertips“, die er bei einer Rede in Las Vegas im November 1994 formulierte, wurde Realität (zit. nach Dernbach 2008).

Einerseits bietet das Netz zahllose Möglichkeiten für öffentliche, alternative Bürgerkommunikation und eine Demokratisierung der digitalen Mediengesellschaft. Jeder und Jede kann heute seine Meinung mit wenig technischem und finanziellem Aufwand in Blogs und Foren online stellen und damit theoretisch weltweit empfangbar sein. Kommentare zu Beiträgen in professionellen Onlinemedien bieten die Möglichkeit der Rückkopplung zu den Autoren und Autorinnen. Über Foren kann Bürgerbeteiligung bei Entscheidungsprozessen auf der kommunalen und regionalen Ebene viel leichter möglich gemacht werden. Bisher schwer organisierbare Interessen können sich leichter vernetzen und an die Öffentlichkeit treten. Protestaktionen wie die „Gelbwesten“ in Frankreich oder die Schülerstreikbewegung „Fridays for Future“ wären ohne die Koordination in sozialen Medien nicht möglich gewesen.

Andererseits finden sich Phänomene wie Desinformation, Lügen, Propaganda, Hasskommentare, Extremismus, Islamismus, Antisemitismus, Neo-Nazismus, Kinderpornografie und andere zweifelhafte oder gar strafbare Inhalte. So lässt sich z. B. nachweisen, dass russische Regierungsstellen während der Corona-Pandemie verstärkt Desinformationen streuen, um das Vertrauen in Gesundheitssysteme und Instrumente für die Krisenbewältigung europäischer Länder zu untergraben. Der Europäische Auswärtige Dienst veröffentlichte, dass in nur zwei Monaten mehr als 150 Fälle von Desinformation aus russischen beziehungsweise prorussischen Quellen registriert wurden (Vertretung der Europäischen Kommission in Deutschland 2020).

Der Rechtsextremist und Antisemit Ken Jebsen gehört mit zur Gruppe der „Corona-Rebellen“ auf YouTube und hat dort 488000 Abonnenten und seine Videos verzeichnen bis zu 700000 Aufrufe. 


\section{Herausforderungen für Demokratie und Politische Bildung}

Die Herausforderungen für die freiheitliche Demokratie westlicher Prägung liegen auf der Hand: Die beschriebene Fragmentierung und der qualitativ neue Strukturwandel der Öffentlichkeit fördert die Verbreitung von Populismus, illiberalen Demokratievorstellungen bis hin zu Diktatur und extremistischen Einstellungen. Damit verbunden ist eine Enttabuisierung bisher geltender zivilisatorischer Standards im gesellschaftlichen Umgang miteinander und die Vernetzung antisozialer, gewaltaffiner Milieus, die bislang keine Öffentlichkeit fanden.

Die Studie „Hass auf Knopfdruck. Rechtsextreme Trollfabriken und das Ökosystem koordinierter Hasskampagnen im Netz“ von Kreißel et al. (2018) belegt die Zunahme von koordiniertem Hass im Netz und dass es sich dabei um eine lautstarke Minderheit handelt. Sie zeigt ferner die Dominanz koordinierter rechtsextremer Accounts. Die pro-AfD-Wahlkampagne mit den Hashtags \#AfD, \#TraudichDeutschland und \#Merkelmussweg im August/September 2017 war die längste und einflussreichste Kampagne der rechtsextremen Troll-Accounts. Diese Kampagnen werden von Reconquista Germanica und verwandten rechtsextremen Onlinenetzwerken aus identitären Kreisen in den sozialen Medien koordiniert, was dazu führt, dass sie teilweise wochenlang in den Toptrends liegen und den Onlinediskurs bestimmen. Die Hashtags werden außerdem von AfD-Accounts und von russischen Medien wie RT und Sputnik aufgegriffen und geraten so in den medialen Mainstream.

Da im digitalen Zeitalter große Öffentlichkeiten direkt und unter Umgehung professioneller Filter und Informationsnavigatoren angesprochen werden können, wird der viralen Verbreitung von Desinformation Vorschub geleistet. Im Folgenden scheint eine leichtere Mobilisierung von Gruppen, eine höhere Volatilität bei Wahlen, eine größere Unberechenbarkeit und Aufsplitterung des Parteiensystems, zunehmende Schwierigkeiten bei der Regierungsbildung durch Koalitionen und damit der Regierbarkeit insgesamt, ein Erstarken von Populismus und Extremismus und ein Verlust von Konsens in der Gesellschaft Realität zu sein (siehe dazu Zick 2016; siehe ebenfalls die anderen „Mitte-Studien“).

Demokratie braucht eine funktionierende Öffentlichkeit. Dazu gehören professionell-journalistische Medien als Navigatoren im zunehmend unübersichtlicher werdenden Informationsdschungel mit entsprechend großen Reichweiten - egal auf welchen Ausspielwegen. Mehr Wissen über Politik durch professionelle Medien kann, muss aber nicht automatisch zu höherem politischem Engagement führen. Deshalb brauchen wir einerseits die Förderung von Medienkompetenz im oben beschriebenen Sinn, um die Rolle der Medien bei der Politikvermittlung 
besser verstehen zu können. Auf der anderen Seite brauchen wir die Stärkung von Demokratiekompetenz zur Gestaltung einer lebendigen demokratischen Kultur. Darunter verstehe ich u. a. mit Himmelmann (2005) sowie Mauz und Gloe (2018) Einstellungen und Werte, praktische Handlungsfertigkeiten und Wissen und kritisches Denken. Diesen drei Bereichen können einzelne Teilkompetenzen zugeordnet werden, die für das Leben in einer Demokratie wichtig sind. Demokratiekompetenz ist das Zusammenkommen aller Teilkompetenzen.

Am Ende muss eine digitale Souveränität stehen, d. h. die Befähigung zur Kultivierung der digitalen Welten. Im Idealfall fließen Medienbildung, Politische Bildung, Soziales Lernen und Werteerziehung zusammen zur digitalen Demokratiekompetenz, d. h. Demokratiekompetenz in einer umfassend und tief greifend mediatisierten und digitalisierten Gesellschaft in einer Welt, die von digitaler Transformation geprägt ist und dauerhaft sein wird. Im Vordergrund von Bildungsbemühungen sollten die Demokratiekompetenzen des Individuums stehen, nicht die digitalen Werkzeuge und Techniken. Digitale Demokratiekompetenz fördern heißt somit, ethisch-kulturelles und politisches Wissen, Reflexionsvermögen sowie Motivation und Wille zum sozialen und politischen Handeln in der digitalisierten Welt zu fördern. Digitale Demokratiekompetenzbildung erfordert für diese neue Aufgabe neue Konzepte und Methoden in der Schnittmenge von politischer, medienpädagogischer, ökonomischer, ethischer und informatischer Bildung. Eine bislang eher getrennte Behandlung der Phänomene gilt es durch inter- und transdisziplinäre Zusammenarbeit zu überwinden. Von besonderer Bedeutung ist hier auch die politische Wertereflexion im Sinne einer „digitalen Ethik“.

Die Förderung von Medienkompetenzen für die digitale Welt sollte kontinuierlich und entlang der Bildungskette - vom Kindergarten über die schulische und außerschulische Jugendbildung bis zur Erwachsenenbildung - erfolgen. Digitale Medienkompetenzförderung ist ein zentraler Bestandteil des lebenslangen Lernens (dazu auch Gapski et al. 2017, S. 26-27). Dazu gehört selbstverständlich auch die systematische Professionalisierung des pädagogischen Personals. Insbesondere die Förderung neuer Querschnittskompetenzen zwischen medienpädagogischer, informationstechnologischer und politischer Bildung sollte für Pädagoginnen und Pädagogen aller Ebenen zu einem verbindlichen Aus- und Fortbildungsziel werden.

Bei der Betonung von Digitalisierung und Mediatisierung darf nicht vergessen und übersehen werden, dass für jede politische Teilhabe und damit auch für die digitale Demokratiekompetenz die kommunikative Kompetenz und insbesondere die klassische Sprachkompetenz von größter Bedeutung bleibt. Sie ist und bleibt grundlegend für kritisch-diskursives Denken. Zentral bleibt die Forderung nach konstruktiv-kreativen und gemeinwohlorientierten Projekten zur Förderung 
digitaler Bildung und Demokratiekompetenz. Sie müssen den häufig an Risiken orientierten und dystopischen Diskursen zur Digitalisierung entgegengestellt werden. Dies kann ein Weg sein, die Herausforderungen für Staat, Gesellschaft und Wirtschaft durch die Digitalisierung zu meistern.

\section{Literatur}

Darmstädter Appell (1995). Aufruf zur Reform der Politischen Bildung in der Schule. Gegenwartskunde, 44, 491-497.

Baacke, D. (1997). Medienpädagogik. Reihe Grundlagen der Medienkommunikation. Band 1. Tübingen: Niemeyer.

Baacke, D. (1999). Medienkompetenz als zentrales Operationsfeld von Projekten. In Dieter Baacke/Susanne Kornblum/Jürgen Lauffer/Lothar Mikos \& Günter A. Thiele (Hrsg.), Handbuch Medien. Medienkompetenz (S. 31-35). Bonn: Bundeszentrale für politische Bildung.

Bruns, A. (2019). Filter bubble. Internet Policy Review, 8(4). https://doi.org/10.14763/2019. 4.1426.

Dernbach, C. (2008, 21. Mai). Transcript of Bill Gates' keynote speech fall/comdex (Nov. 14, 1994) - information at your fingertips (2005) [Blog Post]. https://www.mr-gadget.de/ microsoft/2008-05-21/transcript-of-bill-gates-keynote-speech-fallcomdex-nov-14-1994information-at-your-fingertips-2005.

Detjen, J. (2000). Die Demokratiekompetenz der Bürger. Aus Politik und Zeitgeschichte, 50(25), 11-20.

Detjen, J., Massing, P., Richter, D., \& Weißeno, G. (2012). Politikkompetenz - Ein Modell. Wiesbaden: Springer VS. https://doi.org/10.1007/978-3-658-00785-0.

Dubois, E. \& Blank, G. (2018). The echo chamber is overstated: the moderating effect of political interest and diverse media. In: Information, Communication \& Society 21, S. 729-745.

Fischer, D. (2016, 30. Oktober). Social Bots im US-Wahlkampf. Der Roboter als Wahlkampfhelfer. Der Tagesspiegel. https://www.tagesspiegel.de/gesellschaft/medien/socialbots-im-us-wahlkampf-der-roboter-als-wahlkampfhelfer/14756570.

Friedrich-Ebert-Stiftung. (2019). Neue „Mitte-Studie“: Verlorene Mitte - Feindselige Zustände. Rechtsextreme Einstellungen in Deutschland 2018/19. https://www.fes.de/pre sse/aktuelle-pressehinweise/neue-mitte-studie-verlorene-mitte-feindselige-zustaende-rec htsextreme-einstellungen-in-deutschland-2018-19/.

Gapski, H. (2017). Thesen zu Digitale Demokratiekompetenz. https://www.grimme-forsch ung.de/fileadmin/Grimme_Nutzer_Dateien/Forschung/Dokumente/Thesen_HGapski_D igitale_Demokratiekompetenz-050919.pdf.

Gapski, H., Oberle, M., \& Staufer, W. (2017). Einleitung. In H. Gapski, M. Oberle, \& W. Staufer (Hrsg.), Medienkompetenz. Herausforderung für Politik, politische Bildung und Medienbildung (S. 17-30). Bonn: Bundeszentrale für politische Bildung.

GPJE. (2004). Nationale Bildungsstandards für den Fachunterricht in der Politischen Bildung an Schulen. Ein Entwurf. Schwalbach/Ts.: Wochenschau. 
Himmelmann, G. (2005). Was ist Demokratiekompetenz? Ein Vergleich von Kompetenzmodellen unter Berücksichtigung internationaler Ansätze. Berlin: BLK.

Jüngling, T. (2015, 6. Dezember). Die unheimliche Manipulation durch Robot-Herden. Die Welt. Abgerufen von https://www.welt.de/wirtschaft/webwelt/article149665357/Die-unh eimliche-Manipulation-durch-Robot-Herden.html.

Krammer, R. (2008). Kompetenzen durch Politische Bildung: Ein KompetenzStrukturmodell. Informationen zur Politischen Bildung, 29, 5-14.

Kreißel, P., Ebner, J., Urban, A., \& Guhl, J. (2018). Hass auf Knopfdruck. Rechtsextreme Trollfabriken und das Ökosystem koordinierter Hasskampagnen im Netz. https://www. isdglobal.org/wp-content/uploads/2018/07/ISD_Ich_Bin_Hier_2.pdf.

Kühberger, C. (2009). Kompetenzorientiertes historisches und politisches Lernen. Methodische und didaktische Annäherungen für Geschichte, Sozialkunde und Politische Bildung. Innsbruck: Studienverlag.

Landesmedienanstalten. (2019). MedienGewichtungsstudie 2019. Abgerufen von https:// www.die-medienanstalten.de/fileadmin/user_upload/die_medienanstalten/Themen/For schung/Mediengewichtungsstudie/MedienGewichtungsstudie_2019-2.pdf.

Lobigs, F., \& Neuberger, C. (2018). Meinungsmacht im Internet und die Digitalstrategien von Medienunternehmen. Neue Machtverhältnisse trotz expandierender Internet-Geschäfte der traditionellen Massenmedien-Konzerne. Leipzig: Vistas.

Münchner Manifest (1997). Demokratie braucht politische Bildung: Zum Auftrag der Bundeszentrale und der Landeszentralen für politische Bildung. Aus Politik und Zeitgeschichte, 47(32), 37-38.

Mauz, A., \& Gloe, M. (2018). Demokratiekompetenz bei Service-Learning. Modellentwicklung und Anregungen für die Praxis. Berlin: Stiftung Lernen durch Engagement - Service-Learning in Deutschland.

Media Perspektiven Basisdaten (2019). Daten zur Mediensituation in Deutschland 2019. Frankfurt/M.

Media Perspektiven (2019). Daten zur Mediensituation in Deutschland 2018. Frankfurt a. M..

Schweiger, W. (2017). Der (des)informierte Bürger im Netz. Wie soziale Medien die Meinungsbildung verändern. Wiesbaden: Springer VS. https://doi.org/10.1007/978-3-65816058-6.

Schweiger, W., Weber, P., Prochazka, F., \& Brückner, L. (2019). Algorithmisch personalisierte Nachrichtenkanäle. Begriffe, Nutzung, Wirkung. Wiesbaden: Springer VS. https://doi.org/ https://doi.org/10.1007/978-3-658-24062.

Seemann, M. (2014). Das neue Spiel: Strategien für die Welt nach dem digitalen Kontrollverlust. Freiburg: Orange-Press.

Vertretung der Europäischen Kommission in Deutschland. (2020). Desinformationen zum Coronavirus werden gezielt gestreut. Abgerufen von https://ec.europa.eu/germany/news/ 20200401-bericht-desinformationen-coronavirus_de.

Wallace, J. (2018). Modelling contemporary gatekeeping. Digital Journalism, 6(3), 274-293. https://doi.org/10.1080/21670811.2017.1343648.

Zick, A. (2016). Polarisierung und radikale Abwehr - Fragen an eine gespaltene Gesellschaft und Leitmotive politischer Bildung. In Andreas Zick/Beate Küpper \& Daniela Krause (Hrsg.), Gespaltene Mitte. Feindselige Zustände. Rechtsextreme Einstellungen in Deutschland (S. 203-218). Bonn: Dietz. 
Open Access Dieses Kapitel wird unter der Creative Commons Namensnennung 4.0 International Lizenz (http://creativecommons.org/licenses/by/4.0/deed.de) veröffentlicht, welche die Nutzung, Vervielfältigung, Bearbeitung, Verbreitung und Wiedergabe in jeglichem Medium und Format erlaubt, sofern Sie den/die ursprünglichen Autor(en) und die Quelle ordnungsgemäß nennen, einen Link zur Creative Commons Lizenz beifügen und angeben, ob Änderungen vorgenommen wurden.

Die in diesem Kapitel enthaltenen Bilder und sonstiges Drittmaterial unterliegen ebenfalls der genannten Creative Commons Lizenz, sofern sich aus der Abbildungslegende nichts anderes ergibt. Sofern das betreffende Material nicht unter der genannten Creative Commons Lizenz steht und die betreffende Handlung nicht nach gesetzlichen Vorschriften erlaubt ist, ist für die oben aufgeführten Weiterverwendungen des Materials die Einwilligung des jeweiligen Rechteinhabers einzuholen. 